\title{
Existential meaning as a moderator in stress and psychological health
}

\begin{abstract}
The present study aimed at exploring the mediating role of one's existential meaning in his/her life on Perceived stress and his/her psychological well-being. Victor Frankl's Theoretical paradigm was chosen for this present study. From the University of QuaideAzam, Pakistan a sample of 560 students enrolled in M.Sc, M.Phil \& PhD program was drawn by means of stratified random sampling technique. Existence scale (Langle, Orgler, $\&$ Kundi, 2003), Warwick-Edinburg Mental Well-Being Scale and Stress Scale was used as study instruments. Make use of linear regression is suggestive of the fact that meaning in one's life, the same as affirmed by Frankl moderates the level of stress and psychological well-being.
\end{abstract}

Keywords: existential meaning, stress, psychological health, victor frankl, meaning in life
Volume 4 Issue 5 - 2015

\author{
Aisha Bano,' Rubina Hanif' \\ 'Department of Psychology, Pakistan Medical \& Dental Council, \\ Pakistan \\ ${ }^{2}$ Department of Psychology, National Institute of Psychology, \\ Quaid-e-Azam University, Pakistan
}

\author{
Correspondence: Aisha Bano, Department of Psychology, \\ Pakistan Medical \& Dental Council, Pakistan
} ,Email aisha_latest@hotmail.com

Received: April 09, 2015 | Published: December 29, 2015

\section{Introduction}

Ratni \& Rastogi ${ }^{1}$ (2007) Contends the notion that existential meaning in life can be surmised from the strong spur of the individuals to seek and look for significance in one's life. He further explained that this significance of one's existence is captivated through meaning making process in life. Later they stated it remarkably beneficial from psychic health point of view for looking into one's life meaning and identifying the rationale of one's existence and subsequently sense its significance and importance. It was later furnished through their research that person's who perceives life more meaningful also feel more psychologically in good health. Many recent researches support the fact that looking into one's life's meaning indeed brings several psychic health benefits for thy self. As in a comparative study of the mean scores between individuals with higher and lower meaning in life by Kleftaras \& Psarra $^{2}$ it was indicated that with higher sense of meaning in life the person has a positive sense of psychological health and a less possible probability of developing depressive symptoms.

Adolescents are among those who face real challenges in life with sudden turning points. It had been a real life time challenge for the adolescents to sustain and suffice with their potential personal resources to withstand such stressors successfully. Nielson \& Hansson $^{3}$ study results revealed that adolescents with low meaning in life who were exposed to stress reported illness twice as often as unstressed adolescents did. Thus there had been a growing need to identify those factors that mediate the role in enhancing psychological health and combat stress efficiently. Moreover, a more recent advancement in the studies on adolescents and their reasons for psychological health shows existential meaningfulness as very much fundamental to health protective role in adolescents.

Hypothesis of the study were:

a. Perceived Existential Meaning moderates psychological wellbeing and stress in the study sample.

\section{Method}

Stratified random sampling ensures that there will be selection from each group. For which the 3 already constructed stratas of M.Sc, M.Phil and PhD level classes of university were treated as three separate sub-groups. In the second stage simple random sampling within each stratum was employed for sample selection. In this way a randomized probabilistic sample was selected within each stratum i.e. M.Sc, M.Phil and PhD classes. Sample includes 560 university students out of which the demographic details are as follows: males $=42 \%$, females $=58 \%$; age range is from 19 to 38 years $(\mathrm{M}=22.81$, $\mathrm{SD}=2.71$ ). Existence Scale (Langle, Orgler, Kundi, 2003), WarwickEdinburgh Mental Well-being Scale (Parkinson, 2006) and Stress Scale (Imam, 2006) were used to gather study data on the three variables. After the collection of the data unfinished questionnaires were discarded (total 43) and a total of 560 questionnaires raw scores were loaded onto a spreadsheet for statistical analysis and interpretation. The raw score data which was fed into computer was later "recorded (into same variable)" in the SPSS in order to transform the raw scores into scale's score for the purpose of statistical analysis.

\section{Results}

Results illustrate the alpha coefficient values of Existence Scale, Warwick-Edinburgh Mental Well-Being Scale and Stress Scale to be moderately high for the study sample (Table 1). For study hypothesis linear regression analysis reveals that perceived existential meaning in one's existence was significantly moderates the effects of the two study variables i.e. Psychological well-being and stress; holding accountable for $17 \%$ of variance in psychological well-being and $19 \%$ of the variance in stress (Table $2 \& 3$ ) (Figure $1 \& 2$ ).

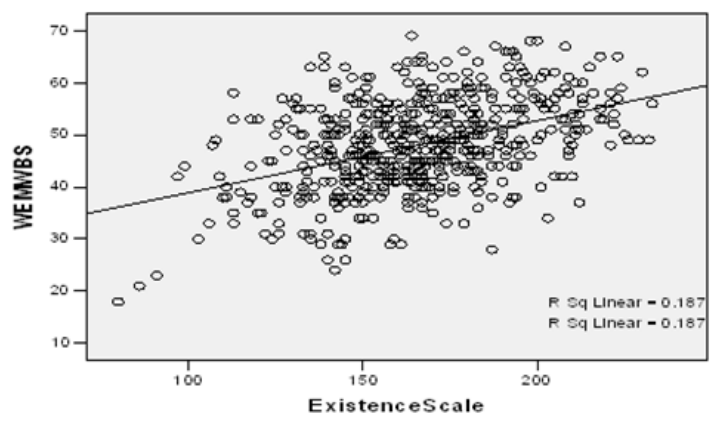

Figure I Linear regression analysis using ES as predictor variable for WEMWBS; $r$ squared linear data $(N=560)$. Note: Vertical axis: variable $y$ -WEMWB Horizontal axis: variable $x$-ES 


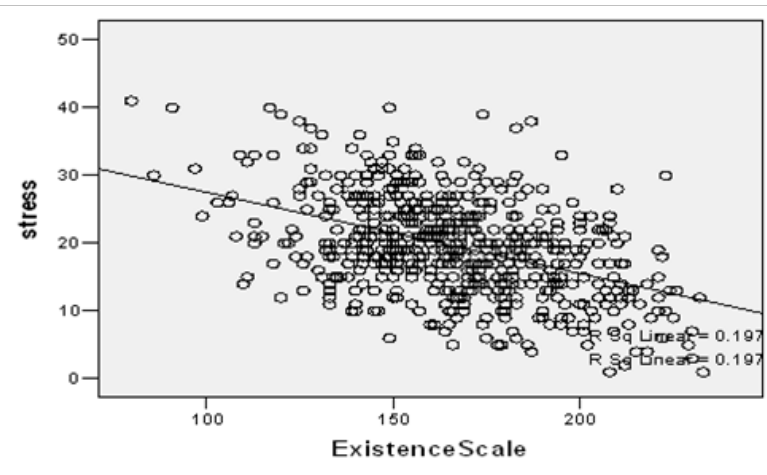

Figure 2 Linear regression analysis using ES as predictor variable for SS; $r$ squared linear data $(N=560)$.

Note: Vertical axis: vertical axis: variable y-SS

Horizontal axis: variable $x$-ES

Table I Descriptive Statistics of Scales (ES,WEMWBS, SS)

\begin{tabular}{ll}
\hline Scales & $\boldsymbol{\alpha}$ \\
\hline Existence Scale & 0.87 \\
Warwick-Edinburgh Mental Well-Being & 0.7 \\
Stress scale & 0.82 \\
\hline
\end{tabular}

Table 2 Linear Regression Analysis using ES as predictor variable for WEMWBS $(\mathrm{N}=560)$

\begin{tabular}{lllll}
\hline Variables & B & SE $\boldsymbol{\beta}$ & B & t \\
\hline Constant & 24.74 & 2.2 & & 11.45 \\
ES & 0.14 & 0.013 & 0.42 & $10.89 * * *$
\end{tabular}

$\mathrm{R} 2=.18$,Adjusted R2 = .17, ${ }^{* *} p<.001$. Note:Please read b as Unstandardized Coefficient; SE as Std Error; and $\beta$ as Std Coefficient

Table 3 Linear Regression Analysis using ES as predictor variable for SS (N $=560$ )

\begin{tabular}{lllll}
\hline Variables & B & SE $\boldsymbol{\beta}$ & B & t \\
\hline Constant & 90.05 & 1.8 & & 22.2 \\
ES & -0.12 & 0.01 & 0.42 & $11.37^{* * * *}$ \\
\hline
\end{tabular}

R2 = .43,Adjusted R2 = .19, *** $\mathrm{p}<.001$ Note. Please read $\mathrm{b}$ as Unstandardized Coefficient; SE as Std Error; and $\beta$ as Std Coefficient

\section{Discussion}

From linear regression analysis it is revealed that the perception of existential meaning in one's life is to a great extent moderating the relationship among the study variables i.e. psychological wellbeing and stress; accounting to explicate $17 \%$ of the variation in one's psychological health and $19 \%$ of the variation in one's stress. This study puts the accent on the importance of the role of the construct "existential meaning in one's life" in one's state of overall well-being. It is interesting to come to know that study results of Clarke et al., ${ }^{4}$ corroborate that those individuals are set on the trail of recuperation enormously faster who engages in setting existential meaning in their recovery process from the illness. Therefore it can be affirmed that the stated prophecy of perceived existential meaning in one's life for being associated with psychological well-being and stress among is thus supported. Implication that it may provide will be a powerful, comprehensive and systematic theoretical guide for further research studies to look into the dynamics of the interplay among different variables i.e. the worthy significance of existential meaning for enhancing one's psychological health and waning the stress.

The study of Brassai et al., ${ }^{5}$ concluded that perceived existential meaning acts as a safeguard against health risk behaviors. So as it is known to every citizen of Pakistan that the uncertainty and the prevailing multi-factorial stress level is increasing day by day within Pakistani culture. It is hence of due estimable importance to find out that through what psychological aspect/facet the practicing psychologist's could counteract the effects of stress and hence to achieve the fundamental goal of enhancing psychological wellbeing. It also may encourage for an action plan by health and fitness practitioners with heavily laid emphasis on the construct of searching for the existential meaning in one's life as affirmed byVictor Frankl. In addition to it the psychic health practitioners may give the longsufferings of mentally ill a kick off by adding or strengthening the meaningfulness component to a person's coping resources. In conclusion the present study is suggestive of the fact that for a hale and hearty societal proliferation and fostering positive mental health in our culture there is a need to inculcate understanding and appraising the need of meaningfulness constituent of one's life.

\section{Acknowledgments}

None.

\section{Conflicts of interest}

Author declares there are no conflicts of interest.

\section{Funding}

None.

\section{References}

1. Neerpal R, Renu R. Meaning in Life and Psychological Well-Being in Preadolescents and Adolescents. Journal of theIndian Academy of Applied Psychology. 2007;33(1):31-38.

2. George K, Evangelia P. Meaning in Life, Psychological Well-Being and Depressive Symptomology: A Comparative Study. Sci Res. 2012;3(4):337-345.

3. Nielsen AM, Hansson K. Associations between adolescents' health, stress and sense of coherence. Stress \& Health. 2007;23(5):331-341.

4. Clarke S, Oades LG, Crowe TP. Recovery in mental health: A movement towards well-being and meaning in contrast to an avoidance of symptoms. Int rev of Psychiatry. 2012;35(4):297-304.

5. Brassai L, Piko BF, Steger MF. Meaning in Life: Is it a protective factor for adolescents' psychological health? Int $J$ Behav Med. 2011;18(1):44-51. 